\title{
LC-ESI-tandem MS and in silico ADMET analysis of polyphenols from Rhus coriaria L. and Micromeria fruticosa (L.) Druce ssp. brachycalyx P. H. Davis
}

\author{
Duygu Taskin ${ }^{1 *}$, Mucahit Ozdemir ${ }^{2}$ and Bahattin Yalcin ${ }^{2}$
}

\begin{abstract}
Background: Micromeria fruticosa (L.) Druce ssp. brachycalyx P. H. Davis and Rhus coriaria L., which are Lamiaceae species, are used both as spices in food and medicinally. Lamiaceae species are known to contain high amounts of polyphenols. In this study, liquid chromatography-quadrupole time-of-flight-tandem mass spectrometry (LC-QTOF-MS/ MS) was used for analysis of polyphenols in the plants. Under gradient elution with using $0.1 \%$ aqueous acetic acid solution and acetonitrile mobile phases, an Agilent Poroshell C18 reversed phase column was used for the simultaneous determination of 18 polyphenols, and separation was performed in 30 min. Pharmacokinetic properties of these polyphenols such as drug-like and toxicity were estimated using open-source software, pkCSM and SwissADME.

Results: These compounds were determined to represent different classes of polyphenols, including phenolic acids, flavonoids, coumarin and tannins. ADMET predictions of polyphenols indicated that these compounds are easily absorbed and do not have toxic effects.

Conclusion: While the Rhus coriaria L. includes anthocyanidins, tannins, phenolic acid and flavonoids, the Micromeria fruticosa (L.) Druce ssp. brachycalyx P. H. Davis has phenolic acid, coumarin and flavonoids, according to these results. In silico ADME/Tox predictions revealed that these bioactive components are to be drug-like and non-mutagenic. These data are supportive for future analysis that can lead to their therapeutic use of the plants, suggesting that this species may be used as a natural medicinal source in the future after detailed analysis tests.
\end{abstract}

Graphical abstract: Keywords: Tandem mass spectrometry, ADMET, Phenolics, Lamiaceae

\section{Background}

Micromeria fruticosa (L.) Druce spp. brachycalyx P.H. (M. fruticosa spp. brachycalyx) is grown in South Anatolia. This species is known as "tas nanesi" and has the smell of peppermint due to its essential oil components. M.fruticosa (Lamiaceae) is widely used in traditional medicine in the form of herbal tea against disorders such as heart diseases, headaches and skin infections.

\footnotetext{
*Correspondence: duygu.taskin@sbu.edu.tr

${ }^{1}$ Department of Analytical Chemistry, Faculty of Pharmacy, University

of Health Sciences, 34668 Istanbul, Turkey

Full list of author information is available at the end of the article
}

Chromatographic (HPLC, GC, GC/MS) studies have shown that the essential oil of $M$. fruticosa contains linalool, pulegone, piperitenone components majorly [1-4].

Rhus coriaria L. (R. coriaria) is grown widely in Africa, South Anatolia, the Mediterranean region and West Asia. It is also common in the Mediterranean and South-eastern of Turkey. This plant, which called sumac, is used as a spice and sauce. It is known that it is traditionally used in diseases such as stomach ailments, hypertension, diuresis and diabetes. It is also known to be used in cancer treatment [5]. It is known that the extracts from the fruits of sumac contain organic acids (malic, 
citric, tartaric fumaric), apigenin neohesperidoside-I, myricetin-glucosides, quercetin-3-O-rhamnoside, tannins and terpenoids [6]. The leaves of the plant have gallic acid, myricetin, quercetin, kaempferol and high levels of tannins [7]. In addition, the essential oil from plants fruits is known to contain carvacrol and $\beta$ caryophyllene, $\alpha$-pinene, cembrene $\alpha$-terpineol [8]. It is known that phenolic compounds, which constitute the widest class of phytochemical compounds contained in both plants, have various bioactive properties. For this reason, it is very important to determine these compounds responsible for the activity in plants qualitatively or quantitatively. When looking through the literature, it is clear that the liquid chromatography-mass spectrometry approach is one of the most widely used methods for analysing phenolic chemicals in medicinal plants $[9,10]$.

The literature review revealed that there were some studies on the content of $R$. coriaria fruit, but no studies on the chemical content of $M$. fruticosa spp. brachycalyx. In addition, the ADMET properties of these phenolic compounds were examined in detail for the first time. Therefore, the primary purpose of this study is to determine the phenolic contents of both plants by LC-QTOFMS/MS. In addition, pharmacokinetic properties such as drug-like and toxicity of polyphenols analysed in plants were estimated for the first time using open-source software, pkCSM and SwissADME.

\section{Methods}

\section{Plant materials and preparation of extracts}

$M$. fruticosa spp. brachycalyx and $R$. coriaria were collected from Kahramanmaraş in Turkey. The identification of the plants has been established by Dr. I. Senkardes from Marmara University, Pharmacy Faculty. The Marmara University herbarium code MARE-19184 and MARE-19185 were assigned to the $M$. fruticosa spp. brachycalyx and $R$. coriaria, respectively.

The aerial parts of plant samples were dried at room temperature and pulverized with a mechanical grinder. The samples $(50 \mathrm{~g})$ were extracted with methanol (for $48 \mathrm{~h} \times 2 ; 400 \mathrm{~mL}$ ) at room temperature. After the solvents were filtered through Whatman No. 1 paper, the filtrate was evaporated to dryness by rotary evaporator (Heidolph Hei-Vap Presicion ML/G1) at $40{ }^{\circ} \mathrm{C}$ and 350 mbar. The raw extracts were kept at $4{ }^{\circ} \mathrm{C}$ in the refrigerator. The extracts $(10 \mathrm{mg})$ were dissolved in $3 \mathrm{~mL}$ of methanolwater solution $(2: 1 \mathrm{v} / \mathrm{v})$. The filtrates were then filtered using $0.2-\mathrm{m}$ Millipore syringe filter, and then $10 \mu \mathrm{L}$ samples were injected to LC system.

\section{LC-ESI-Tandem MS analysis}

The polyphenolic compounds of the extracts from aerial parts of plants were determined by LC-ESI-tandem MS technique. An Agilent 6530 was used to separate and analyse polyphenolic compounds. The chromatographic separation was performed on reverse phase Agilent Poroshell C18 $(3 \times 150 \mathrm{~mm}, 2.7 \mu \mathrm{m})$ analytical column. The column temperature was set to $30^{\circ} \mathrm{C}$. The separation was carried with a gradient binary mixture of solvent $\mathrm{A}$ ( $0.1 \%$ aqueous acetic acid) and solvent $B(0.1 \%$ acetic acid acetonitrile) at a flow rate of $0.4 \mathrm{~mL} / \mathrm{min}: 0-5 \mathrm{~min} 10 \% \mathrm{~B}$; $2-5 \min 10-50 \% \mathrm{~B} ; 5-9$ min $50 \% \mathrm{~B} ; 9-10 \min 50-90 \%$ B; 10-12 min $50-90 \%$ B; $12-18$ min $10-90 \%$ B; $18-25$ min $10-90 \% \mathrm{~B} ; 25-25.01 \mathrm{~min} 90-10 \% \mathrm{~B}$ and stop time is $30.00 \mathrm{~min}$. The full mass and fragmentation spectra of the polyphenols were generated by the electrospray ionization with quadrupole time-of-flight analyser in negative ion mode. The use of helium as collision gas and nitrogen was used as nebulizing gas.

\section{ADMET prediction}

The ADMET word is an abbreviation for absorption, distribution, metabolism, excretion and toxicity. ADMET studies, one of the cheminformatics computers programs, provide us with very important data on whether a chemical compound can be used as a medicine or not without conducting experimental studies. In this study, pkCSM, a free online web server (http://structure.bioc. cam.ac.uk/pkcsm) [11], was used to predict the pharmacological properties of compounds from $R$. coriaria and $M$. fruticosa spp. brachycalyx. Pharmaceutical values (lipophilicity, size, polarity, insolubility, insaturation and flexibility) of compounds and their radar charts were produced using SwissADME (http://www.swissadme.ch) [12]. The ADMET properties of 18 compounds which extracted from R. coriaria and M. fruticosa spp. brachycalyx were calculated by computer, and the partition coefficients $(\log \mathrm{P})$ of all compounds in this study were found.

\section{Results}

\section{Chromatographic separation}

LC-ESI-tandem MS analysis was used to qualitatively analyse of polyphenolic compounds in $R$. coriaria and $M$. fruticosa spp. brachycalyx (Figs. 1 and 2). Major polyphenolic compounds were analysed by comparing the molecular weights of these compounds and their fragments with the fragments provided by standard compounds and literature information. The analysis of gallic, syringic, quinic and caffeic acid, and rutin in the LC MS system was analysed with authentic standards by comparing their molecular weights, retention times and the mass/ charge ratios of the fragment ions they gave after fragmentation. The other polyphenols were also tentatively identified by LC-QTOF-MS/MS with negative ionization because lacking reference standards. By comparing 


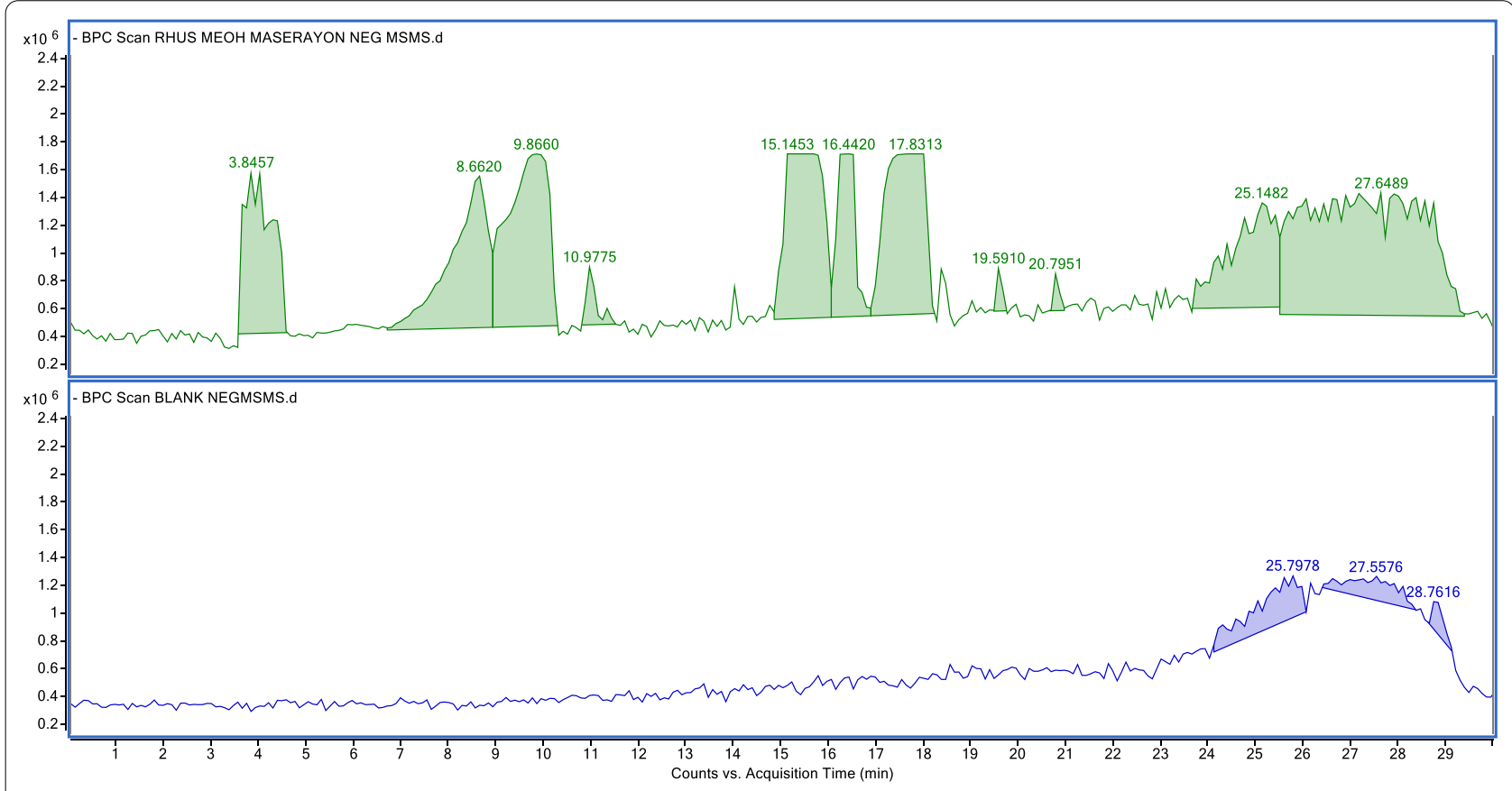

Fig. 1 MS base peak and blank chromatogram of R. coriaria methanol extract

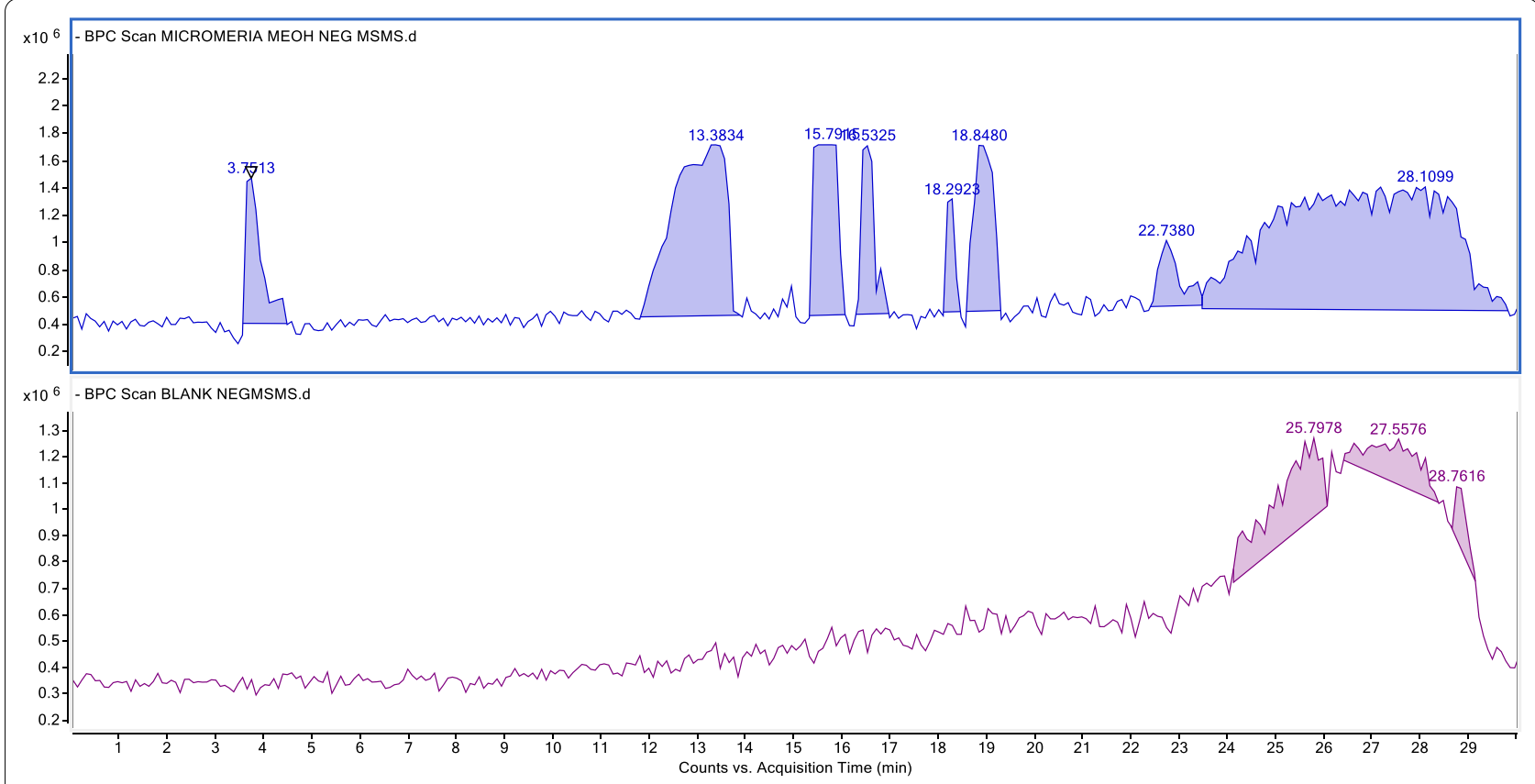

Fig. 2 MS base peak and blank chromatogram of M. fruticosa spp. brachycalyx methanol extract 
Table 1 Identification of polyphenols in R. coriaria by LC-ESI-tandem MS data

\begin{tabular}{|c|c|c|c|c|}
\hline$R t$ (Min) & {$[\mathrm{M}-\mathrm{H}]^{-}$} & Other MS-MS ions (M/Z) & Tentative identification & References \\
\hline 3.9634 & 331.0747 & $313,271,241,211,169,151,123,89,71,59$ & Galloyl-hexoside & [13] \\
\hline 3.9293 & 655.1924 & $493,433,331,311,271,169,89,59$ & Malvidin-3,5-O-diglucoside & [15] \\
\hline 4.0052 & 523.1408 & $331,271,191,169,123,59$ & Galloyl-hexoside-hexoside & [13] \\
\hline 8.6787 & 663.1473 & $493,331,271,169,59$ & $\begin{array}{l}\text { Galloyl-hexoside + galloyl-hexoside } \\
{[\mathbf{2 M}-\mathbf{H}]^{-}}\end{array}$ & [13] \\
\hline 8.7205 & 493.1328 & $436,313,241,211,169,123,89,59,39$ & Galloyl-di-O-hexoside & [14] \\
\hline 10.1689 & 169.0157 & $125,97,79,69,51,41,25$ & Gallic acid & * \\
\hline 13.8988 & 359.1020 & $327,299,239,197,182,169,153,123,89,59,44$ & Syringic acid-O-hexoside & [16] \\
\hline 17.3932 & 197.0470 & $169,124,106,78,69,53,32$ & Syringic acid & * \\
\hline 20.5924 & 301.0310 & $283,255,223,191,165,149,138,107,65$ & Tricetin & [17] \\
\hline
\end{tabular}

* Compounds identified by comparing retention times and MS data with those of reference compounds

Table 2 Identification of polyphenols in M. fruticosa spp. brachycalyx by LC-ESI-tandem MS data

\begin{tabular}{|c|c|c|c|c|}
\hline$R t$ (min) & {$[\mathrm{M}-\mathrm{H}]^{-}$} & Other MS-MS ions $(\mathrm{m} / \mathrm{z})$ & Tentative identification & References \\
\hline 3.7679 & 683.2282 & $341,251,179,89$ & Hexose polymer & [18] \\
\hline 12.7601 & 353.8721 & $265,191,161,135,111,85,44$ & 5-O-caffeoylquinic acid & [19] \\
\hline 12.9454 & 191.0565 & $171,127,111,93,85,67,59,44$ & Quinic acid & * \\
\hline 13.0547 & 179.0359 & $152,135,107,89,71,59,41$ & Caffeic acid & * \\
\hline 15.6230 & 353.0936 & $335,271,173,135,93,43$ & Shikimoyl-hexose & {$[20]$} \\
\hline 16.6920 & 221.0484 & $203,177,159,148,133,115,77,55$ & Derivative of methoxy coumarin & {$[21]$} \\
\hline 18.2498 & 461.0753 & $417,323,285,221,161,113,44$ & Kaempferol glucuronide & [22] \\
\hline 18.3257 & 609.1546 & $300,271,151$ & Rutin & * \\
\hline 18.4434 & 439.1450 & $395,330,221,161,133,89,59$ & Malonyl-monocqa & [23] \\
\hline
\end{tabular}

"Compounds identified by comparing retention times and MS data with those of reference compounds

spectra of compounds that have previously recorded mass fragmentation patterns in full scan mode (MS) and MS/MS modes, the compounds' identities were confirmed in the literature (Additional file 1).

In this study, it was analysed that the methanol extract from aerial parts mainly contained phenolic acids, flavonoids, coumarin and tannins. The molecular weights and fragments of these compounds are shown in Tables 1 and 2.

When the results in Table 1 were evaluated, it was found that galloyl hexoside dimer was formed in at $8.67 \mathrm{~min} \mathrm{[13].} \mathrm{The} \mathrm{formation} \mathrm{of} \mathrm{dimeric} \mathrm{moieties} \mathrm{is} \mathrm{a}$ common situation in LC-MS/MS studies and is associated with the geometric structures of molecules. Galloyl hexoside comes in at $3.96 \mathrm{~min}$ and eludes after the dimer formation $(8.67 \mathrm{~min})$. Galloyl hexoside and dihexoside [14] have been formed in at 4.00 and $8.72 \mathrm{~min}$, respectively. The sugar portion was separated and gave a fragment of gallate at a molecular weight of $170 \mathrm{~g} / \mathrm{mol}$. MS/ MS fragments show us an anthocyanin aglycone and sugar molecule in at $3.9293 \mathrm{~min}$ as malvidin-3,5-Odiglucoside [15]. It gives 655.19 a molecule that has broken a proton. By separating the glucose part from the molecule, a fragment ion of 331 molecular mass was formed, which is in the structure of malvidin. The molecular ion peak gives $[\mathrm{M}-\mathrm{H}]^{-}$ion at $\mathrm{m} / z 169.0157$ as a gallic acid, and fragment ion peak gives at $\mathrm{m} / \mathrm{z} 125$ ([gallic acid- $\left.\mathrm{H}-\mathrm{CO}_{2}\right]^{-}$). Syringic acid-O-hexoside was formed in at $13.89 \mathrm{~min}$ [16]. The aglycone as well as the presence of the $\mathrm{O}$-hexoside moiety was proven by the $[\mathrm{M}-\mathrm{H}-162]^{-}$ $\mathrm{m} / z 197$ ion and by the $m / z 182$ [syringic acid- $\left.\mathrm{H}-\mathrm{CH}_{3}\right]^{-}$, $\mathrm{m} / z 169$ [syringic acid $\left.-2 \mathrm{CH}_{3}\right]^{-}$and $\mathrm{m} / z 153$ [syringic acid- $\left.\mathrm{H}-\mathrm{CO}_{2}\right]^{-}$ions. Also, aglycon syringic acid was formed in at 17.39 min with $[\mathrm{M}-\mathrm{H}]^{-}$at $m / z$ 197. Based on the comparison of their $\mathrm{MS}^{2}$ spectra with reported literature, base peak which gave $[\mathrm{M}-\mathrm{H}]^{-}$value at $\mathrm{m} / \mathrm{z}$ 301.0310 was tentatively identified as tricetin [17].

When the results in Table 2 were evaluated, it was determined that molecular ion peak gives $[\mathrm{M}-\mathrm{H}]^{-}$ion at $\mathrm{m} / z 683.2282$ as a hexose polymer as the literature [18]. Caffeic acid showed $[\mathrm{M}-\mathrm{H}]^{-}$value at $m / z$ 179.0329. The fragment ions at $m / z 161$ and 135 by losses of a $\mathrm{H}_{2} \mathrm{O}$ molecule and a $\mathrm{CO}_{2}$ molecule were found, respectively. Rutin showed that $[\mathrm{M}-\mathrm{H}]^{-}$value at $m / z 609.2080$ gave product ions at $m / z 301$ by losses rutinose. The molecular ion peak gives $[\mathrm{M}-\mathrm{H}]^{-}$ion at $m / z 353.8721$ as a 5-O-caffeoylquinic 
Table 3 The ADMET parameters of polyphenols from $R$. coriaria via pkcsm software

\begin{tabular}{|c|c|c|c|c|c|c|c|c|c|}
\hline & $\begin{array}{l}\text { Galloyl } \\
\text { hexoside }\end{array}$ & $\begin{array}{l}\text { Malvidin- } \\
\text { 3,5- } \\
\text { diglucoside }\end{array}$ & $\begin{array}{l}\text { Galloyl } \\
\text { dihexoside }\end{array}$ & Syringic acid & $\begin{array}{l}\text { Galloyl- } \\
\text { di-O- } \\
\text { hexoside }\end{array}$ & Gallic acid & Tricetin & $\begin{array}{l}\text { Syringic } \\
\text { acid-O- } \\
\text { hexoside }\end{array}$ & Quinic acid \\
\hline \multicolumn{10}{|l|}{ Absorption } \\
\hline $\begin{array}{l}\text { Water solubility } \\
(\log \mathrm{mol} / \mathrm{L})\end{array}$ & -1.89 & -2.866 & -2.705 & -2.223 & -2.895 & -2.56 & -3.028 & -2.501 & -0.911 \\
\hline $\begin{array}{l}\text { Caco2 perme- } \\
\text { ability }\left(\log P_{c}\right. \\
\mathrm{cm} / \mathrm{s})\end{array}$ & -0.795 & -1.345 & -0.866 & 0.495 & -1.682 & -0.081 & -0.272 & -0.485 & -0.418 \\
\hline $\begin{array}{l}\text { Intestinal } \\
\text { absorption } \\
(\% \mathrm{~A})\end{array}$ & 37.36 & 0 & 0 & 73.08 & 15.64 & 43.37 & 78.37 & 25.25 & 21.667 \\
\hline $\begin{array}{l}\text { Skin Permeabil- } \\
\text { ity (log Kp) }\end{array}$ & -2.735 & -2.735 & -2.735 & -2.735 & -2.735 & -2.735 & -2.735 & -2.735 & -2.735 \\
\hline $\begin{array}{l}\text { P-glycoprotein } \\
\text { substrate }\end{array}$ & No & Yes & Yes & Yes & Yes & No & Yes & Yes & No \\
\hline $\begin{array}{l}\text { P-glycoprotein I } \\
\text { inhibitor }\end{array}$ & No & No & No & No & No & No & No & No & No \\
\hline $\begin{array}{l}\text { P-glycoprotein II } \\
\text { inhibitor }\end{array}$ & No & No & No & No & No & No & No & No & No \\
\hline \multicolumn{10}{|l|}{ Distribution } \\
\hline$V_{D s s}{ }^{a}$ & 0.517 & 1.205 & -0.058 & -1.443 & 1.614 & -1.855 & 0.932 & -0.782 & -0.817 \\
\hline $\begin{array}{l}\text { Fraction } \\
\text { unbound }\end{array}$ & 0.818 & 0.247 & 0.428 & 0.601 & 0.347 & 0.617 & 0.208 & 0.645 & 0.737 \\
\hline $\begin{array}{l}\text { BBB } \\
\text { permeability } \\
\text { (log BB) }\end{array}$ & -1.616 & -2.459 & -1.665 & -0.191 & -2.435 & -1.102 & -1.38 & -1.434 & -1.085 \\
\hline $\begin{array}{l}\text { CNS } \\
\text { permeability } \\
\text { (log PS) }\end{array}$ & -4.465 & -5.358 & -7.028 & -2.701 & -4.668 & -3.74 & -3.557 & -4.147 & -4.399 \\
\hline \multicolumn{10}{|l|}{ Metabolism } \\
\hline $\begin{array}{l}\text { CYP2D6 sub- } \\
\text { strate }\end{array}$ & No & No & No & No & No & No & No & No & Yes \\
\hline $\begin{array}{l}\text { CYP3A4 sub- } \\
\text { strate }\end{array}$ & No & No & No & No & No & No & No & No & No \\
\hline $\begin{array}{l}\text { CYP1A2 inhibi- } \\
\text { tor }\end{array}$ & No & No & No & No & No & No & Yes & No & No \\
\hline $\begin{array}{l}\text { CYP2C19 inhibi- } \\
\text { tor }\end{array}$ & No & No & No & No & No & No & No & No & No \\
\hline $\begin{array}{l}\text { CYP2C9 inhibi- } \\
\text { tor }\end{array}$ & No & No & No & No & No & No & No & No & No \\
\hline $\begin{array}{l}\text { CYP2D6 inhibi- } \\
\text { tor }\end{array}$ & No & No & No & No & No & No & No & No & No \\
\hline $\begin{array}{l}\text { CYP3A4 inhibi- } \\
\text { tor }\end{array}$ & No & No & No & No & No & No & No & No & No \\
\hline \multicolumn{10}{|l|}{ Excretion } \\
\hline $\begin{array}{l}\text { Total clearance } \\
\text { (log } \mathrm{ml} / \mathrm{min} / \\
\mathrm{kg})\end{array}$ & 0.512 & -0.077 & 0.535 & 0.646 & 0.47 & 0.518 & 0.513 & 0.646 & 0.639 \\
\hline $\begin{array}{c}\text { Renal OCT2 } \\
\text { substrate }\end{array}$ & No & No & No & No & No & No & No & No & No \\
\hline \multicolumn{10}{|l|}{ Toxicity } \\
\hline AMES toxicity & No & No & No & No & No & No & No & No & No \\
\hline $\begin{array}{l}\text { Maximum toler- } \\
\text { ated dose } \mathrm{d}^{\mathrm{d}}\end{array}$ & 0.22 & 0.468 & 0.2 & 1.374 & 0.453 & 0.7 & 0.545 & 1.168 & 2.148 \\
\hline hERG I inhibitor & No & No & No & No & No & No & No & No & No \\
\hline hERG II inhibitor & No & Yes & Yes & No & Yes & No & No & No & No \\
\hline
\end{tabular}


Table 3 (continued)

\begin{tabular}{|c|c|c|c|c|c|c|c|c|c|}
\hline & $\begin{array}{l}\text { Galloyl } \\
\text { hexoside }\end{array}$ & $\begin{array}{l}\text { Malvidin- } \\
\text { 3,5- } \\
\text { diglucoside }\end{array}$ & $\begin{array}{l}\text { Galloyl } \\
\text { dihexoside }\end{array}$ & Syringic acid & $\begin{array}{l}\text { Galloyl- } \\
\text { di-O- } \\
\text { hexoside }\end{array}$ & Gallic acid & Tricetin & $\begin{array}{l}\text { Syringic } \\
\text { acid-O- } \\
\text { hexoside }\end{array}$ & Quinic acid \\
\hline $\begin{array}{l}\text { Oral rat acute } \\
\text { Toxicity }\end{array}$ & 2.414 & 2.5 & 2.493 & 2.157 & 2.505 & 2.218 & 2.421 & 2.389 & 1.539 \\
\hline $\begin{array}{l}\text { Oral rat chronic } \\
\text { Toxicity }\end{array}$ & 4.092 & 4.843 & 5.701 & 2.415 & 4.675 & 3.06 & 2.551 & 3.718 & 3.433 \\
\hline Hepatotoxicity & No & No & No & No & No & No & No & Yes & No \\
\hline $\begin{array}{l}\text { Skin Sensitiza- } \\
\text { tion }\end{array}$ & No & No & No & No & No & No & No & No & No \\
\hline $\begin{array}{l}\text { T. Pyriformis } \\
\text { toxicity (log } \\
\mu \mathrm{g} / \mathrm{L} \text { ) }\end{array}$ & 0.285 & 0.285 & 0.285 & 0.281 & 0.285 & 0.285 & 0.31 & 0.285 & 0.285 \\
\hline $\begin{array}{l}\text { Minnow toxicity } \\
(\log m M)\end{array}$ & 6.856 & 8.253 & 6.19 & 2.554 & 8.255 & 3.188 & 4.09 & 6.404 & 3.812 \\
\hline
\end{tabular}

${ }^{a}$ Volume of Distribution (log L/kg)

${ }^{b}$ BBB (Blood-brain barrier)

${ }^{c}$ CNS (Central nervous system)

${ }^{\mathrm{d}}$ Maximum tolerated dose unit is (log $\mathrm{mg} / \mathrm{kg} /$ day)

e Oral rat acute toxicity unit is $(\mathrm{mol} / \mathrm{kg})$ and these values are lethal dose, $50 \%\left(\mathrm{LD}_{50}\right)$

${ }^{\mathrm{f}}$ Oral rat chronic toxicity unit is (log $\mathrm{mg} / \mathrm{kg} \mathrm{bw/day)}$

acid as the literature [19]. The compound in at $16.69 \mathrm{~min}$ is thought to be a methoxy coumarin in the light of the relevant literature [21]. Deprotonated molecular ions at $m / z 191$ and fragment ions at $m / z 173\left[\mathrm{M}-\mathrm{H}-\mathrm{H}_{2} \mathrm{O}\right]^{-}$, $127\left[\mathrm{M}-\mathrm{H}-\mathrm{H}_{2} \mathrm{O}-\mathrm{H}_{2} \mathrm{O}-\mathrm{CO}\right]^{-}$were generated during the peak 12.95th minute. As a result, it was characterized as quinic acid. The compound in at $18.25 \mathrm{~min}$ had the $[\mathrm{M}-\mathrm{H}]^{-}$ion at $m / z 461$ which yielded the fragment ion at $m / z 285\left([\mathrm{M}-\mathrm{H}]^{-}-176\right.$, loss of one glucuronyl unit). As a result, kaempferol monoglucuronide was tentatively identified [22]. Malonyl-mono caffeoylquinic acid (malonyl-monocqa) was tentatively identified in at $18.44 \mathrm{~min}$ based on comparison of their $\mathrm{MS}^{2}$ spectra with reported literature [23].

\section{In silico ADMET profiling of phenolic compounds from plant}

The pharmacokinetics of compounds were predicted by the parameters of absorption, distribution, metabolism, excretion and toxicity as shown in Tables 3 and 4. The Caco-2 permeability values of all compounds were predicted to be low. Galloyl hexoside, syringic acid, gallic acid, tricetin, hexose polymer, 5-O-caffeoylquinic acid, caffeic acid and 6,8-dimethoxy-7-hydroxycoumarin were predicted to have high absorbed the intestinal absorption (human). All compounds were not predicted to be permeable skin. Galloyl hexoside, gallic acid, hexose polymer, caffeic acid and 6,8-dimethoxy-7-hydroxycoumarin were predicted to have not P-glycoprotein substrate. All of compounds were predicted to have not inhibitory effects. Galloyl hexoside, malvidin-3,5-diglucoside, galloyl-di-O-hexoside, tricetin, 5-O-caffeoylquinic acid, kaempferol-3-O-glucuronide and rutin had high the volume of distribution. All compounds were predicted to be poorly distributed to the blood-brain barrier, and they unable to penetrate the Central Nervous System. It is estimated that p450 enzymes, mostly found in the liver, do not metabolize the analysed compounds. It is also predicted that the same molecules are not substrates for this enzyme. It is estimated that no analyses compounds are a substrate for organic cation transport protein 2 . Not all compounds analysed are predicted to have mutagenic and minnow toxicity effects. Syringic acid-O-hexoside was predicted to be hepatotoxicity effect. None of the compounds were predicted to have the potential to inhibit hERG I. However, malvidin-3,5-diglucoside, gallic acid, galloyl-di-O-hexoside and rutin have hERG II inhibitory effects. None of the compounds were predicted to have skin sensitization. When the log P values of all compounds are examined, it is estimated that the molecules except tricetin, caffeic acid, syringic acid and dimethoxyhydroxycoumarin are more hydrophilic. It can be seen from the values in Tables 3 and 4 that these four molecules with more lipophilic properties are absorbed more easily too.

The relationship between drug permeability and lipophilicity in brain capillaries is shown in Fig. 3 [24]. As can be seen in the figure, it is estimated that the uptake of many substances into the brain will be limited due to their low octanol/water distribution coefficient. Among 
Table 4 The ADMET parameters of polyphenols from M. fruticosa spp. brachycalyx via pkcsm software

\begin{tabular}{|c|c|c|c|c|c|c|c|c|}
\hline & $\begin{array}{l}\text { Hexose } \\
\text { polymer }\end{array}$ & $\begin{array}{l}\text { 5-O-Caffeoylquinic } \\
\text { acid }\end{array}$ & Caffeic acid & $\begin{array}{l}\text { Kaempferol-3- } \\
\text { O-glucuronide }\end{array}$ & Rutin & $\begin{array}{l}\text { Malonyl- } \\
\text { caffeoyl- } \\
\text { quinic acid }\end{array}$ & $\begin{array}{l}\text { 6,8-Dimethoxy-7- } \\
\text { hydroxycoumarin }\end{array}$ & $\begin{array}{l}\text { Shikimoyl- } \\
\text { hexose }\end{array}$ \\
\hline \multicolumn{9}{|l|}{ Absorption } \\
\hline $\begin{array}{l}\text { Water solubility } \\
(\log \mathrm{mol} / \mathrm{L})\end{array}$ & -1.381 & -2.449 & -2.33 & -2.866 & -2.892 & -2.965 & -2.458 & -0.214 \\
\hline $\begin{array}{l}\text { Caco2 perme- } \\
\text { ability (log } P_{c} \\
\mathrm{~cm} / \mathrm{s})\end{array}$ & -0.359 & -0.84 & 0.634 & -0.884 & -0.949 & -0.744 & 0.378 & -0.481 \\
\hline $\begin{array}{l}\text { Intestinal } \\
\text { absorption } \\
(\% \mathrm{~A})\end{array}$ & 30.68 & 36.38 & 69.41 & 25.17 & 23.45 & 8.338 & 95.59 & 6.657 \\
\hline $\begin{array}{l}\text { Skin permeabil- } \\
\text { ity (log Kp) }\end{array}$ & -2.913 & -2.735 & -2.722 & -2.735 & -2.735 & -2.735 & -2.945 & -2.747 \\
\hline $\begin{array}{l}\text { P-glycoprotein } \\
\text { substrate }\end{array}$ & No & Yes & No & Yes & Yes & Yes & No & Yes \\
\hline $\begin{array}{l}\text { P-glycoprotein I } \\
\text { inhibitor }\end{array}$ & No & No & No & No & No & No & No & No \\
\hline $\begin{array}{l}\text { P-glycoprotein II } \\
\text { inhibitor }\end{array}$ & No & No & No & No & No & No & No & No \\
\hline \multicolumn{9}{|l|}{ Distribution } \\
\hline$V_{D s s}^{a}$ & -0.069 & 0.581 & -1.098 & 1.295 & 1.663 & 0.147 & -0.354 & 0.283 \\
\hline $\begin{array}{l}\text { Fraction } \\
\text { unbound }\end{array}$ & 0.891 & 0.658 & 0.529 & 0.28 & 0.187 & 0.43 & 0.316 & 0.663 \\
\hline $\begin{array}{l}\text { BBB } \\
\text { permeability } \\
\text { (log BB) }\end{array}$ & -0.895 & -1.407 & -0.647 & -1.441 & -1.899 & -2.069 & -0.377 & -1.051 \\
\hline $\begin{array}{l}\text { CNS } \\
\text { permeability } \\
\text { (log PS) }\end{array}$ & -3.359 & -3.856 & -2.608 & -3.955 & -5.178 & -3.71 & -2.473 & -5.681 \\
\hline \multicolumn{9}{|l|}{ Metabolism } \\
\hline $\begin{array}{l}\text { CYP2D6 sub- } \\
\text { strate }\end{array}$ & No & No & No & No & No & No & No & No \\
\hline $\begin{array}{l}\text { CYP3A4 sub- } \\
\text { strate }\end{array}$ & No & No & No & No & No & No & No & No \\
\hline $\begin{array}{l}\text { CYP1A2 inhibi- } \\
\text { tor }\end{array}$ & No & No & No & No & No & No & Yes & No \\
\hline $\begin{array}{l}\text { CYP2C19 inhibi- } \\
\text { tor }\end{array}$ & No & No & No & No & No & No & No & No \\
\hline $\begin{array}{l}\text { CYP2C9 inhibi- } \\
\text { tor }\end{array}$ & No & No & No & No & No & No & No & No \\
\hline $\begin{array}{l}\text { CYP2D6 inhibi- } \\
\text { tor }\end{array}$ & No & No & No & No & No & No & No & No \\
\hline CYP3A4inhibitor & No & No & No & No & No & No & No & No \\
\hline \multicolumn{9}{|l|}{ Excretion } \\
\hline $\begin{array}{l}\text { Total clearance } \\
\text { (log ml/min/ } \\
\text { kg) }\end{array}$ & 0.907 & 0.307 & 0.508 & 0.503 & -0.369 & -0.036 & 0.713 & 1.524 \\
\hline $\begin{array}{c}\text { Renal OCT2 } \\
\text { substrate }\end{array}$ & No & No & No & No & No & No & No & No \\
\hline \multicolumn{9}{|l|}{ Toxicity } \\
\hline AMES toxicity & No & No & No & No & No & No & No & No \\
\hline $\begin{array}{l}\text { Max. tolerated } \\
\text { dose (log mg/ } \\
\text { kg/day) }\end{array}$ & 1.865 & -0.134 & 1.145 & 0.46 & 0.452 & 1.029 & 0.56 & 1.208 \\
\hline hERG I inhibitor & No & No & No & No & No & No & No & No \\
\hline hERG II inhibitor & No & No & No & No & Yes & No & No & No \\
\hline
\end{tabular}


Table 4 (continued)

\begin{tabular}{|c|c|c|c|c|c|c|c|c|}
\hline & $\begin{array}{l}\text { Hexose } \\
\text { polymer }\end{array}$ & $\begin{array}{l}\text { 5-O-Caffeoylquinic } \\
\text { acid }\end{array}$ & Caffeic acid & $\begin{array}{l}\text { Kaempferol-3- } \\
\text { O-glucuronide }\end{array}$ & Rutin & $\begin{array}{l}\text { Malonyl- } \\
\text { caffeoyl- } \\
\text { quinic acid }\end{array}$ & $\begin{array}{l}\text { 6,8-Dimethoxy-7- } \\
\text { hydroxycoumarin }\end{array}$ & $\begin{array}{l}\text { Shikimoyl- } \\
\text { hexose }\end{array}$ \\
\hline $\begin{array}{l}\text { Oral rat acute } \\
\text { Toxicity }\end{array}$ & 0.955 & 1.973 & 2.383 & 2.513 & 2.491 & 2.389 & 2.326 & 1.958 \\
\hline $\begin{array}{l}\text { Oral rat chronic } \\
\text { Toxicity }\end{array}$ & 3.553 & 2.982 & 2.092 & 4.641 & 3.673 & 3.756 & 1.825 & 4.068 \\
\hline Hepatotoxicity & No & No & No & No & No & No & No & No \\
\hline $\begin{array}{l}\text { Skin sensitiza- } \\
\text { tion }\end{array}$ & No & No & No & No & No & No & No & No \\
\hline $\begin{array}{l}\text { T. Pyriformis } \\
\text { toxicity (log } \\
\mu \mathrm{g} / \mathrm{L})\end{array}$ & 0.285 & 0.285 & 0.293 & 0.285 & 0.285 & 0.285 & 0.431 & 0.285 \\
\hline $\begin{array}{l}\text { Minnow toxicity } \\
(\log m M)\end{array}$ & 5.494 & 5.741 & 2.246 & 6.898 & 7.677 & 5.661 & 1.862 & 5.541 \\
\hline
\end{tabular}

${ }^{\text {a }}$ Volume of Distribution (log $\mathrm{L} / \mathrm{kg}$ )

${ }^{b}$ BBB (blood-brain barrier)

${ }^{c}$ CNS (central nervous system)

${ }^{d}$ Oral rat acute toxicity unit is $(\mathrm{mol} / \mathrm{kg})$ and these values are lethal dose, $50 \%\left(\mathrm{LD}_{50}\right)$

e Oral rat chronic toxicity unit is (log $\mathrm{mg} / \mathrm{kg}$ bw/day)

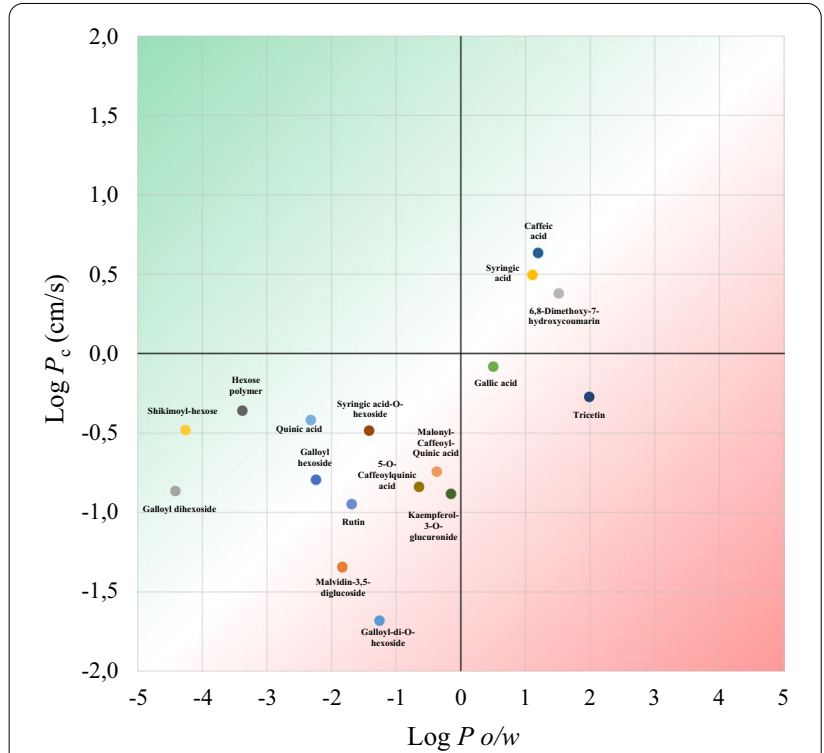

Fig. 3 Drug permeability in brain capillaries $\left(\log p_{c}\right)$ as a function of partition coefficient $(\log p \circ / w)$

the compounds, caffeic and syringic acid and methoxy coumarin derivatives are predicted to be more hydrophobic, so they can pass the brain barrier more easily than the others.

The biggest problem that can be encountered in oral administration of a drug molecule is bioavailability. The bioavailability radar device is used for oral bioavailability estimation, such as lipophilicity, size, polarity, solubility, flexibility, and saturation to determine drug affinity. In Fig. 4, some pharmacological properties of the compounds from M. fruticosa spp. brachycalyx and R. coriaria were predicted by SwissADME. Figure 4 presents an oral bioavailability radar field based on the lipophilicity, molecular size, polarity, TPSA and water solubility criteria of 18 compounds analysed in plants. As shown in the figure, syringic acid, hexose polymer and dimethoxyhydroxycoumarin are predicted to have suitable physicochemical profiles for oral bioavailability. However, polar values of ten other molecules and saturation values of three ones indicate that they fall outside the desired range for bioavailability.

\section{Discussion}

In one a previous study, phenolic content of water and ethanol extract of $R$. coriaria was analysed by LC-MS/ MS and flavonoid, phenolic acid and galloyl compounds [25] were determined. In this study, we started our research with the knowledge that the biological activity results made with this plant are stronger in the methanol extract [4], and therefore, we conducted tandem MS analysis of the methanol extract of the plant. In contrast to the above-mentioned study, we analysed the sugary structures of malvidin, syringic acid and galloyl compounds, syringic acid aglycon and tricetin in $R$. coriaria. There is also a publication on the leaf of R. coriaria [26]. In this publication, the authors determined that this species contains galloyl compounds. As a result of our tandem MS studies, it was proven that this species contains galloyl compounds as well as some phenolic acids and 


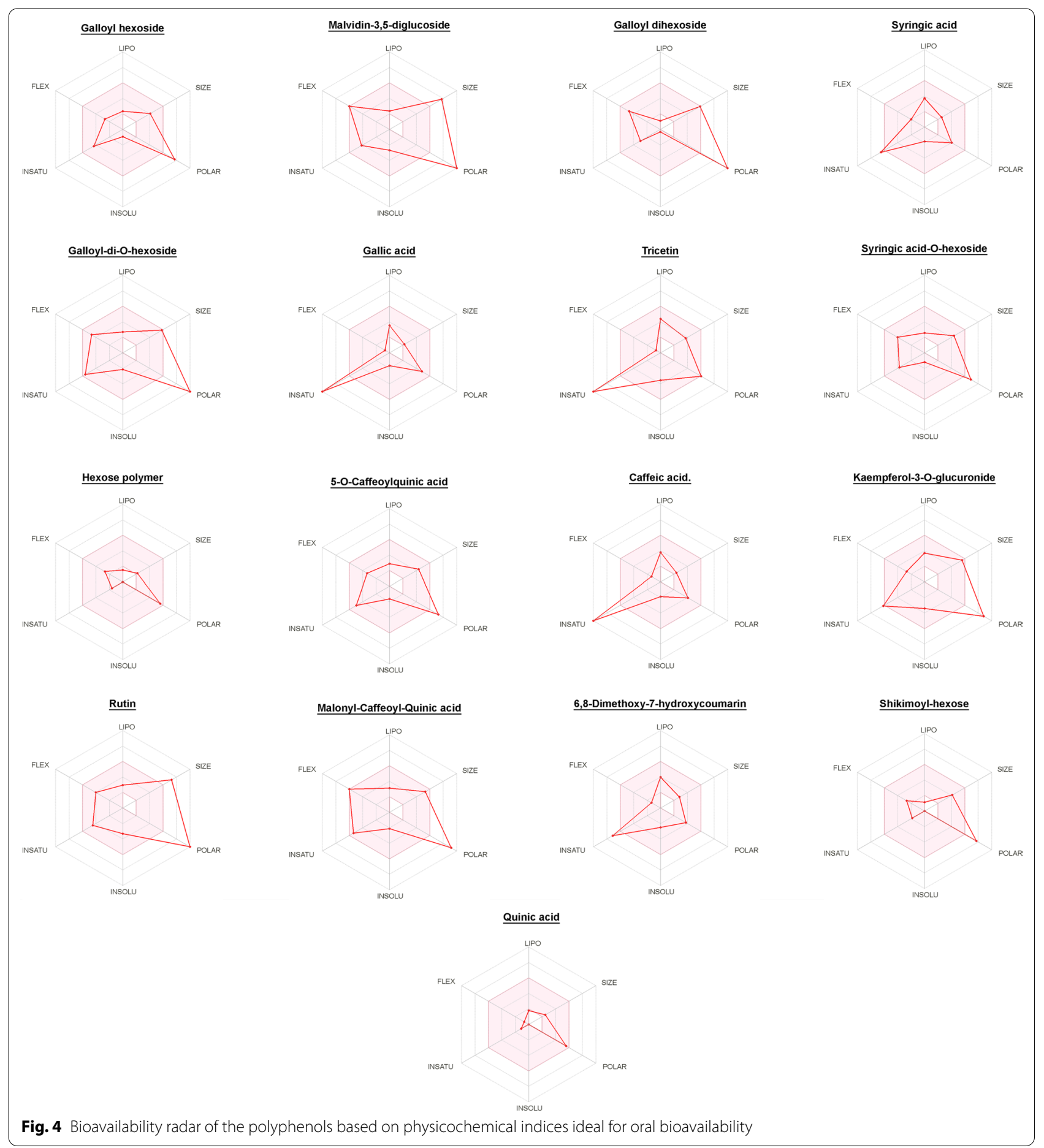

tricetin. As shown in Table 1, a total of nine compounds were analysed in $R$. coriaria; seven compounds were analysed tentatively based on literature information, while the other two compounds were analysed based on spectral and chromatographic information given by standard substances.

In a study, leaf parts of $M$. fruticosa L. extracted with $80 \%$ methanol and the phytometabolites in the extract were widely analysed in untargeted mode by LC-MS/ 
MS and a total of 215 compounds were identified tentatively [27]. In another study, phenolic compounds of $M$. graeca (L.) Benth. ex Rchb were analysed by HPLC [28]. In another study, alpha amylase and tyrosinase enzyme inhibition and antioxidant activity of different extracts of $M$. nervosa were studied and the phenolic compounds in its content were analysed by LC-MS/MS [29]. As can be understood from the studies in the literature, there are very limited studies on the phenolic compound analysis on Micromeria species. Phenolic compounds of M. fruticosa spp. brachycalyx were analysed by us for the first time in tandem MS. As can be seen in Table 2, a total of nine compounds were analysed in the plant; six compounds were analysed tentatively in the light of literature information, while the other three compounds were analysed in the light of spectral and chromatographic information given by standard substances.

In a study, methanol extracts of Syzygium cumini (black plum) seeds and Allium cepa (onion) peels were analysed by GC MS and it was found that there were 20 phytocomponents in the plant, and four of these compounds had drug-like pharmacokinetic properties [30]. There are publications in the literature on the pharmacokinetic properties of the natural compounds using the in silico ADMET process [31-34]. Therefore, evaluation of the in silico ADME properties of biologically active compounds before in vivo and clinical studies in drug or drug raw material design and development based on a plant would be a great time saving and a good data source. The ADMET predictions of phenolic compounds show that some of these compounds are easily absorbed and do not have toxic effects, suggesting that this species can be used as a natural medicinal and nutritional source in the future after detailed analyses. The hydrophilic or hydrophobic properties of the compounds analysed in the chromatography system are shown with octanol-water partition coefficients. These partition coefficients are related to the distribution of compounds in the body. The high coefficient indicates that the molecule is hydrophobic and dispersed into the hydrophobic areas of the cell, while the low coefficient indicates that the molecule is hydrophilic and can be dispersed into aqueous areas such as blood serum [35].

While only a small fraction of lipophilic substances can cross the blood-brain barrier, most polar and hydrophilic substances cannot be transported to the brain without a special delivery system [36]. Among the compounds, caffeic and syringic acid and methoxy coumarin derivatives are predicted to be more hydrophobic, so they can pass the brain barrier more easily. Besides, the bioactive compound of the plants was predicted to be drug-like and non-mutagenic with in silico ADMET. These data support data for future analysis that can lead to their therapeutic use of the plants.

\section{Conclusions}

As a result, in this study, the phytochemical contents of these two species, which are used by the public for food and medical purposes, were analysed with tandem MS. According to the findings obtained, totally eighteen phenolic compounds, which of nine were analysed in $R$. coriaria and the other nine in the M. fruticosa spp. brachycalyx, were tentatively identified based on MS determination and fragmentation pattern. As a result of chromatographic analysis, it was determined that $R$. coriaria is rich in tannins and $M$. fruticosa spp. brachycalyx is rich in phenolic acid and flavonoid. Besides, the ADMET properties of these phenolic compounds contained in these two species were estimated in silico. The fact that some compounds in both plants are well absorbed, have drug-like properties and do not have toxic effects supports the traditional use of these two species.

\section{Abbreviations}

LC-ESI: Tandem MS liquid chromatography-electrospray ionization tandem mass spectroscopy; ADMET: Absorption, distribution, metabolism, excretion and toxicity; HPLC: High-performance liquid chromatography; GC/MS: Gas chromatography-mass spectroscopy.

\section{Supplementary Information}

The online version contains supplementary material available at https://doi. org/10.1186/s43094-021-00317-0.

Additional file 1. LC-ESI-Tandem MS spectra fragmentation patterns of polyphenols.

Acknowledgements

Authors are highly thankful to Dr. İsmail Şenkardeş, for identified the plants.

\section{Authors' contributions}

We declare that this study was conducted by the authors named in this article. DT performed the tandem MS analysis, interpreting ADMET results and writing the article, MÖ performed the in silico ADMET analysis, BY supervise the work. All the authors read and approved the final manuscript.

Funding

Proposed work not funded by any funding agencies.

Availability of data and materials

All data and material are available upon request.

\section{Declarations}

Ethics approval and consent to participate Not applicable.

Consent for Publication

Not applicable.

Competing interests

The authors declare no competing interest. 


\section{Author details}

${ }^{1}$ Department of Analytical Chemistry, Faculty of Pharmacy, University of Health Sciences, 34668 Istanbul, Turkey. ${ }^{2}$ Department of Chemistry, Faculty of Science, Marmara University, 34722 Istanbul, Turkey.

\section{Received: 5 March 2021 Accepted: 10 August 2021}

Published online: 26 August 2021

\section{References}

1. Slavkovska V, Couladis M, Bojovic S, Tzakou O, Pavlovic M, Lakusic B, Jancic R (2005) Essential oil and its systematic significance in species of Micromeria Bentham from Serbia \& Montenegro. Plant Systemat Evol 255:1-15

2. Telci I, Ceylan M (2007) Essential oil composition of Micromeria fruticosa Druce from Turkey. Chem Nat Compd 43(5):629-631

3. Martin E, Cetin Ö, Dirmenci T, Ay H (2011) Karyological studies of Clinopodium L. (Sect. Pseudomelissa) and Micromeria Benth. S. str. (Lamiaceae) from Turkey. Caryologia 64:398-404

4. Taskin T, Dogan M, Yilmaz BN, Senkardes I (2020) Phytochemical screening and evaluation of antioxidant, enzyme inhibition, anti-proliferative and calcium oxalate anti-crystallization activities of Micromeria fruticosa spp. brachycalyx and Rhus coriaria. Biocatal Agric Biotechnol 27:1-7

5. Kossah R, Nsabimana C, Zhao J, Chen H, Tian F, Zhang H, Chen W (2009) Comparative study on the chemical composition of Syrian sumac (Rhus coriaria L.) and Chinese sumac (Rhus typhina L.) fruits. Pak J Nutr 8(10):1570-1574

6. Bursal E, Köksal E (2011) Evaluation of reducing power and radical scavenging activities of water and ethanol extracts from sumac (Rhus coriaria L.). Food Res Int 44(7):2217-2221

7. Asgarpanah J, Saati S (2014) An overview on phytochemical and pharmacological properties of Rhus coriaria L. R JP 1(3):47-54

8. Abu-Reidah IM, Ali-Shtayeh MS, Jamous RM, Arráez-Román D, SeguraCarretero A (2015) HPLC-DAD-ESI-MS/MS screening of bioactive components from Rhus coriaria L. (Sumac) fruits. Food Chem 166:179-191

9. Sulaiman CT, Ramesh PR, Mahesh K, Madhu KM, Anandan EM, Praveen M, Balachandran I (2020) Chemical profiling of a polyherbal formulation by tandem mass spectroscopic analysis with multiple ionization techniques. FJPS 6:40

10. Liu Y, Liu J, Wang Y, Abozeid A, Tang ZH (2016) Simultaneous determination of six active metabolites in Astragalus mongholicus (Fisch.) Bge. under salt stress by ultra-pressure liquid chromatography with tandem mass spectrometry. Springer Plus 5:927

11. Pires DE, Blundell TL, Ascher DB (2015) pkCSM: predicting small-molecule pharmacokinetic and toxicity properties using graph-based signatures. J Med Chem 58(9):4066-4072

12. Daina A, Michielin O, Zoete V (2017) SwissADME: a free web tool to evaluate pharmacokinetics, drug-likeness and medicinal chemistry friendliness of small molecules. Sci Rep 7(1):1-13

13. Mena P, Calani L, Dall'Asta C, Galaverna G, García-Viguera C, Bruni R, Crozier A, Del Rio D (2012) Rapid and comprehensive evaluation of (poly) phenolic compounds in pomegranate (Punica granatum L.) juice by UHPLC-MSn. Molecules 17(12):14821-14840

14. Mekam PN, Martini S, Nguefack J, Tagliazucchi D, Stefani E (2019) Phenolic compounds profile of water and ethanol extracts of Euphorbia hirta L. leaves showing antioxidant and antifungal properties. S Afr J Bot 127:319-332

15. Lantzouraki DZ, Sinanoglou VJ, Tsiaka T, Proestos C, Zoumpoulakis P (2015) Total phenolic content, antioxidant capacity and phytochemical profiling of grape and pomegranate wines. RSC Adv 5:101683-101692

16. Hofmann T, Nebehaj E, Alber L (2016) Antioxidant properties and detailed polyphenol profiling of European hornbeam (Carpinus betulus L.) leaves by multiple antioxidant capacity assays and high-performance liquid chromatography/multistage electrospray mass spectrometry. Ind Crops Prod 87:340-349
17. William K, Price E, Ashton J, Tapsell LC, Johnson S (2016) Identification and characterization of phenolic compounds in hydromethanolic extracts of sorghum wholegrains by LC-ESI-MS ${ }^{n}$. Food Chem 211:215-226

18. Martinez L, Spinola EJ, Sandra VG (2015) HPLC-ESI-MSn characterization of phenolic compounds, terpenoid saponins, and other minor compounds in Bituminaria bituminosa. Ind Crops Prod 69:80-90

19. Li Y, Liu Y, Liu R, Liu S, Zhang X, Wang Z, Zhang J, Lu J (2015) HPLC-LTQorbitrap MSn profiling method to comprehensively characterize multiple chemical constituents in xiao-er-qing-jie granules. Anal Methods 7:7511

20. Fraternalea D, Riccia D, Verardob G, Gorassinic A, Stocchia V, Sestilia P (2015) Activity of Vitis vinifera tendrils extract against phytopathogenic fungi. Nat Prod Commun 10:1037-1042

21. Terraza PS, Villarroya AL, Fourcroy P, Briat JF, Abadía A, Gaymard F, Abadía J, Fernández AA (2016) Accumulation and secretion of coumarinolignans and other coumarins in Arabidopsis thaliana roots in response to iron deficiency at high Ph. Front Plant Sci 23:7-1711

22. Goufo P, Singh RK, Cortez I (2020) A reference list of phenolic compounds (including stilbenes) in grapevine (Vitis vinifera L.) roots, woods, canes, stems, and leaves. Antioxidants (Basel) 9(5):398

23. Zhang Y, Shi P, Qu H, Cheng Y (2007) Characterization of phenolic compounds in Erigeron breviscapus by liquid chromatography coupled to electrospray ionization mass spectrometry. Rapid Commun Mass Spectrom 21(18):2971-2984

24. Bodor N, Buchwald P (1999) Recent advances in the brain targeting of neuropharmaceuticals by chemical delivery systems. Adv Drug Deliv Rev 36(2-3):229-254

25. Tohma H, Altay A, Köksal E et al (2019) Measurement of anticancer, antidiabetic and anticholinergic properties of sumac (Rhus coriaria): analysis of its phenolic compounds by LC-MS/MS. Food Measure 13:1607-1619

26. Beretta G, Rossoni G, Santagati NA, Facino RM (2009) Anti-ischemic activity and endothelium-dependent vasorelaxant effect of hydrolysable tannins from the leaves of Rhus coriaria (Sumac) in isolated rabbit heart and thoracic aorta. Planta Med 75(14):1482-1488

27. Abu-Reidah IM, Arráez-Román D, Al-Nuri M, Warad I, Segura-Carretero A (2018) Untargeted metabolite profiling and phytochemical analysis of Micromeria fruticosa L. (Lamiaceae) leaves. Food Chem 279:128-143

28. Brahmi F, Guendouze N, Hauchard D, Okusa P, Kamagaju L, Madani K, Duez P (2017) Phenolic profile and biological activities of Micromeriagraeca (L.) Benth. ex Rchb. Int J Food Prop 20:2070-2083

29. Sarikurkcu C, Andrade JC, Ozer MS, de Lima Silva JMF, Ceylan O, de Sousa EO et al (2020) LC-MS/MS profiles and interrelationships between the enzyme inhibition activity, total phenolic content and antioxidant potential of Micromeria nervosa extracts. Food Chem 328:126930

30. Kadri HS, Minocheherhomji FP (2020) ADMET analysis of phyto-components of Syzygium cumini seeds and Allium cepa peels. FJPS 6:117

31. Durán-Iturbide NA, Díaz-Eufracio BI, Medina-Franco JL (2020) In silico ADME/Tox profiling of natural products: a focus on BIOFACQUIM. ACS Omega 5:16076-16084

32. Ntie-Kang F, Lifongo LL, Mbah JA, Owono Owono LC, Megnassan E, Mbaze LM, Judson PN, Sippl W, Efange SMN (2013) In silico drug metabolism and pharmacokinetic profiles of natural products from medicinal plants in the Congo basin. Silico Pharmacol 1:12

33. Yi F, Li L, Xu LJ et al (2018) In silico approach in reveal traditional medicine plants pharmacological material basis. Chin Med 13:33

34. Perumal PC, Sowmya S, Pratibha P et al (2015) Isolation, structural characterization and in silico drug-like properties prediction of a natural compound from the ethanolic extract of Cayratia trifolia (L.). Pharmacogn Res 7(1):121-125

35. Shargel L, Susanna WP, Yu AB (2012) Physiological drug distribution and protein binding. Appl Biopharm Pharmacokinet 6:211

36. Çetin M, Çapan Y (2004) Brain drug targeting. J Fac Pharm 33(4):287-305

\section{Publisher's Note}

Springer Nature remains neutral with regard to jurisdictional claims in published maps and institutional affiliations. 